\title{
Premature ovarian insufficiency: the context of long-term effects
}

\author{
A. Podfigurna-Stopa ${ }^{1}$ A. Czyzyk $^{1} \cdot$ M. Grymowicz ${ }^{2} \cdot$ R. Smolarczyk ${ }^{2} \cdot$ K. Katulski ${ }^{1}$. \\ K. Czajkowski ${ }^{3} \cdot$ B. Meczekalski ${ }^{1}$
}

Received: 6 February 2016 / Accepted: 4 April 2016 / Published online: 18 April 2016

(C) The Author(s) 2016. This article is published with open access at Springerlink.com

\begin{abstract}
Purpose Premature ovarian insufficiency (POI) is defined as the cessation of the ovarian function before the age of 40 years. POI aetiology may be related to iatrogenic or endogenous factors and in many cases remains unclear. The aim of this review was to characterize the long-term consequences of POI.

Methods The available literature regarding the long-term consequences of POI from MEDLINE has been reviewed.

Results Lack of ovarian steroids synthesis has serious consequences for women's health. The short-term effects are similar to spontaneous menopause and refer mainly to the climacteric syndrome. In a longer perspective, POI affects a variety of aspects. It obviously and drastically reduces the chances for spontaneous pregnancies. Oestrogen loss leads also to urogenital atrophy. The most common urogenital symptoms include vaginal dryness, vaginal irritation and itching. The urogenital atrophy and hypoestrogenism interferes also with sexual functioning. Patients with POI are threatened by a decrease in bone mineral density (BMD). POI women also experience psychological distress and some studies have shown an increased risk of neurodegenerating diseases. Overall, POI women have a shortened life expectancy, mainly due to cardiovascular
\end{abstract}

B. Meczekalski

blazejmeczekalski@yahoo.com

1 Department of Gynecological Endocrinology, Poznan University of Medical Sciences, ul. Polna 33, Poznan, Poland

2 Department of Gynecological Endocrinology, Warsaw Medical University, Warsaw, Poland

3 II Department of Obstetrics and Gynaecology, Warsaw Medical University, Warsaw, Poland disease. Some studies have reported a reduced risk of breast cancer in this group of patients.

Conclusions In conclusion there are several well-characterized health risks in POI women. With every patient, an individualized approach is required to properly recognize and prevent these risks.

Keywords Premature ovarian insufficiency .

Cardiovascular disease $\cdot$ Hypoestrogenism - Bone mineral density $\cdot$ Sexual dysfunction $\cdot$ Fertility

\section{Introduction}

Premature ovarian insufficiency (POI) is defined as an ovarian insufficiency before the age of 40 years [1]. It is characterized by a cessation of menstruation for at least 4 months associated with the elevation of serum follicle-stimulating hormone (FSH) concentration (FSH >40 IU/l) [2]. Development of hypergonadotropic hypogonadism before the age of 40 affects approximately $1-2 \%$ of women [3].

Premature ovarian insufficiency can have an influence on premature morbidity and mortality [4]. It is related to profound hypoestrogenism, which has a deleterious effect on different systems in the female body [1]. For the first time, this condition was described by Fuller Albright in 1942 and defined as primary ovarian insufficiency [5].

POI can have a spontaneous or induced background. Spontaneous POI is referred to genetic, autoimmunological, inflammatory, enzyme deficiency, metabolic, or very often idiopathic causes [6, 7]. Induced POI occurs mainly due to oncological treatment such as surgery (bilateral oophorectomy), chemotherapy and radiotherapy [6]. The following factors have been associated with POI: heredity factors, ovarian surgery, pelvic surgery, chemotherapy and 


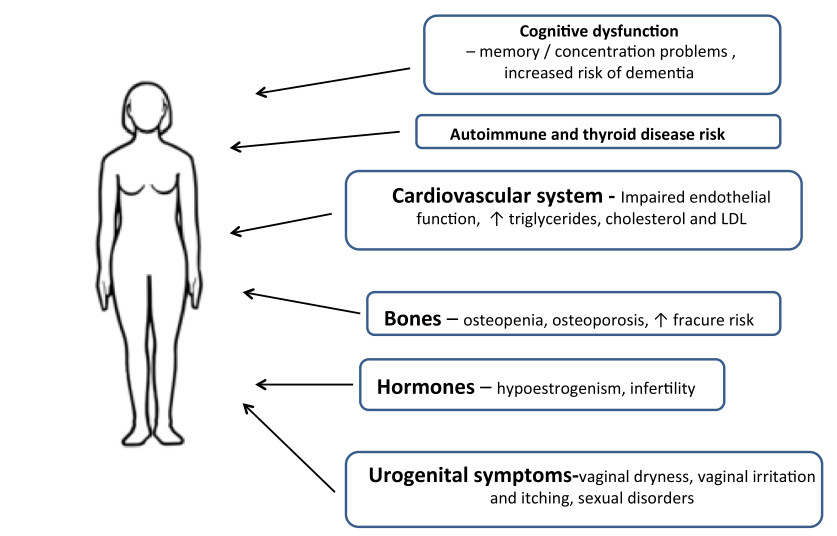

Mortality- increased risk of premature death

Fig. 1 Long-term consequences of premature ovarian insufficiencyschematic summary

radiotherapy, exposure to toxicity and smoking, irregular menses in the history $[8,9]$.

The consequences of POI can be divided into short and long-term consequences.

POI short-term consequences mostly result from prompt oestrogen deficiency. Short-term consequences may include vasomotor symptoms like hot flushes, night sweats, heart palpitations or headaches. The long-term health risks of POI are infertility, osteoporosis, cardiovascular and neurologic diseases and an increased risk of premature death [10]. Women with the diagnosis of POI also present psychological problems including irritability, forgetfulness, insomnia and poor concentration [6]. Moreover, women with POI may present vaginal atrophy, which is responsible very often for dyspareunia. The aim of this review was to summarize the available scientific data regarding POI long-term consequences (Fig. 1).

\section{Fertility, pregnancy, pregnancy outcome}

Twenty five percent of POI cases has intermittent and unpredictable course, and the chance for spontaneous conception has been estimated to $4-10 \%[11,12]$. The ovarian reserve of such women is assessed by an ultrasound count of antral follicles and serum anti-mullerian hormone $(\mathrm{AMH}), \mathrm{FSH}$, estradiol (E2), and inhibin B (InhB) measurements. A cohort of 358 patients with idiopathic POI followed up for almost 6 years was described by Bidet et al. [12]. The cumulative pregnancy course was $4.3 \%$ at 48 months. Twenty-one spontaneous pregnancies resulted in 16 live births, one twin birth, four miscarriages and one elective abortion [12]. Two pregnancies were complicated by the occurrence of gestational diabetes mellitus, one by hypothyroidism, and one of the singleton pregnancies resulted in a preterm birth.
Little progress has been made to improve reproduction with patients' own gametes. There are several studies evaluating the effectiveness of oestrogen, Gonadotropin-releasing hormone $(\mathrm{GnRH})$ analogues, glucocorticoids or danazol pretreatment continued with ovarian stimulation with gonadotropins. Ovulation is achieved in approximately $20 \%$ of POI patients. However, pregnancy rates for most of the strategies, assessed in systematic review by Robles et al. [13], were similar to the spontaneous pregnancy rates for these patients.

Oocyte donation is the only proven and recommended treatment for women with POI. The pregnancy rate after an oocyte donation cycle is around $40 \%$ [14]. Cumulative pregnancy rates of oocyte donation treatment are very high and after four cycles reach 70-80\% [14]. Ameratunga et al. [15] reported following complications in 36 pregnancies after oocyte donation in POI patients: three cases of preterm labour, five gestational hypertensions, two gestational diabetes mellitus and one intrauterine growth retardation case. There were two twin gestations after a 2-embryo transfer.

There is an overall increase in cancer prevalence followed by an increase in long-term survival of the affected patients. Protection against iatrogenic POI caused by chemotherapy, radiation therapy or surgery assumes a high priority. Shielding or ovarian transposition during radiotherapy and fertility sparing surgery should be considered in young females undergoing cancer treatment. GnRH analogue given during chemotherapy significantly reduces the risk of POI in young cancer patients, but does not exhibit its protective effects in fertility $[16,17]$.

Cryopreservation of embryos and mature oocytes is the clinically established method, with pregnancy rates and livebirths reaching $25 \%[18,19]$.

All remaining options, such as retrieving immature oocytes aiming at maturing them later in vitro, freezing of gonadal tissue or novel methods using ovarian stem cells, are very promising but still considered experimental.

\section{Urogenital symptoms}

Oestrogen loss leads to urogenital atrophy. The most common urogenital symptoms include vaginal dryness, vaginal irritation and itching [20]. The symptoms have been widely studied in women undergoing age-appropriate menopausal transition. However, there are few studies concerning the prevalence and treatment of genitourinary syndrome in POI patients. The frequency of most menopausal symptoms in the spontaneous POI group is generally lower than in medically induced premature menopause, with the exception of sexual function. It is worsened in more than half of affected patients in both groups [21]. 
The study evaluating the vaginal flora and the vaginal trophism of 36 women with POI under the age of 40 years, using hormonal therapy, found no significant differences regarding cytological aspects, $\mathrm{pH}$ measurements, vaginal microflora types and fungi infection comparing to agematched women with normal gonadal function [22]. However, when sexual function was evaluated in both groups, women with POI showed worse sexual performance, with more pain and poorer lubrication than women in the control group [23]. These findings suggest that hormonal therapy in POI patients successfully reestablishes the epithelium cells, vaginal $\mathrm{pH}$ and microflora, but is not effective enough to improve complaints of lubrication and pain.

There are no studies reporting a higher occurrence of urinary incontinence among POI patients. Six POI women were studied urodynamically before and after the administration of oral and vaginal oestrogen. Oestrogen supplementation did not produce any significant changes in the functional or cystometric parameters. The authors concluded that oestrogen alone is of minimal significance in maintaining normal urinary tract physiology in the absence of ageing and other factors leading to urinary incontinence [24].

\section{Cancer risk}

Cancer risk in POI patients is a complex issue. First, ovarian reserve depletion may occur as a result of cytotoxic cancer therapy in prepubertal or childbearing age patients and there is always concern about the cancer recurrence or subsequent neoplasms risk. Next, long-term hormonal treatment may carry additional cancer risk in subgroups of POI patients. Moreover, there are some epidemiological studies suggesting that the early cessation of ovarian function may be an independent cancer mortality risk factor.

There is no evidence that oestrogen replacement in spontaneous POI increases the risk of breast cancer in comparison with normally menstruating women [25]. Therefore, hormone therapy is recommended until the average age of the natural menopause and there is no need to start mammographic screening early [2]. It is, however, suggested that progestagens with the lower potential risk should be preferentially used [12]. Hormonal therapy with oestrogen and progestagens is regarded as contraindicated in breast cancer survivors [26]. Adolescent patients experiencing acute ovarian failure after cancer treatment will not go through puberty without oestrogen treatment [27]. However, in the subsets of childhood cancer survivors, such as Hodgkin disease survivors with the greater risk of breast cancer, heart disease and stroke, hormonal therapy implementation should be individualized [28].
A large study of middle-age and elderly Chinese women demonstrated that POI was inversely associated with the incidence of breast cancer [29]. This phenomenon is explained by the fact that the cumulative life-exposure of women with POI to endogenous circulating sex hormones is shorter than that of women with later menopause, leading to a reduced risk of breast cancer over a woman's lifetime. Nevertheless, in the same study, POI has been associated with an increased risk of cancer mortality, and this increase was independent of hormonal replacement therapy [31].

\section{Cardiovascular disease}

The main reason for shortened life expectancy in POI patients is cardiovascular disease and, therefore, some studies have addressed the issue of cardiovascular risk in this group of women. To date it has been shown that POI women present several risk factors for the development of cardiovascular disease: endothelial dysfunction, autonomic dysfunction, abnormal lipid profile, insulin action disturbances and metabolic syndrome.

Endothelial function, measured as the flow-mediated dilation of the brachial artery, has been shown to be significantly reduced in POI women. Similarly, the number of circulating endothelial progenitor cells number is diminished and correlated with a decreased serum estradiol concentration [30, 31]. The POI women present an increased carotid intima media thickness and left ventricular diastolic function [33]. Interestingly, the hormonal therapy of 6 months duration is able to improve the flow-mediated dilation by 2.4 -fold, to the same levels as in healthy controls [32]. Goldemeier et al. [32] have also displayed normal endothelial-dependent vasodilation in POI women under hormonal therapy. Despite this, in the same study the authors showed impaired baroreflex sensitivity $(3.9 \pm 1.38$ vs. $7.15 \pm 3.62 \mathrm{~ms} / \mathrm{mmHg}$ ) and reduced heart rate variability $\left(2310 \pm 1173\right.$ vs. $\left.3754 \pm 1921 \mathrm{~ms}^{2}\right)$ of POI women $(N=17)$ in comparison to healthy controls $(N=15)$ [34].

POI patients present abnormalities in lipid profile, but the results are conflicting regarding particular lipoproteins. As Knauff et al. [33] have reported, POI women $(N=90)$ show significantly higher TG levels (mean difference: $0.17 \log \mathrm{mmol} / \mathrm{L}$ [95 \% CI 0.06-0.29]) and lower HDL cholesterol levels in comparison to controls $(n=198)$ after correction for age, body mass index and smoking. This difference has not been confirmed in a smaller study $(N=47$ POI vs. 60 controls) by Gulhan et al. [34]. However, this group revealed significantly higher TC and LDL levels in POI patients and a significant negative correlation between E2 and TC levels $(r=-0.291, P=0.047)$. Recently, Ates et al. [36] reported increased TC and HDL cholesterol in POI women $(N=59)$ vs. healthy controls $(N=59)$. The 
analysed population presented similar levels of glucose, insulin, HOMA-IR, low-density lipoprotein cholesterol (LDL-C), and triglyceride as the controls, but the incidence of metabolic syndrome was significantly increased [35]. In contrast, other authors detected increased serum glucose, insulin and the homeostasis model of assessment-insulin resistance (HOMA-IR) in POI women $(N=43)$ vs. controls $(N=33)$ [36].

Even though there are conflicting data regarding lipid profile and insulin resistance indices, the overall cardiovascular risk in POI women seems to be significantly increased, as the mortality causes analyses in this group has shown (above). Especially the risk of mortality from ischaemic heart disease is approximately $80 \%$ increased in the POI women group compared to women with menopause at 49-55 years [37].

\section{Bone mineral density}

An association between oestrogen deficiency in the postmenopausal period and osteoporosis has been clearly established (North American Menopause Society) [38]. Albright et al. [39] publications are the first to demonstrate the relationship between oestrogen deficiency, menopause and an increased incidence of fractures in women [41].

However, in young women hypoestrogenism and hypoandrogenemia have a deleterious effect on peak bone mass (PBM) formation and bone mineral density (BMD) status [40]. Lana et al. [41] found that serum FSH concentrations, but not oestradiol, are positively associated with bone mass loss in skeletal regions (both the spinal column and femoral neck) in patients with spontaneous POI.

Numerous studies have revealed a significant decrease of BMD in POI patients. Uygur et al. [42] found that both the femoral neck bone and spinal bone BMD were significantly lower in POI patients than measurements of the control group.

A large study conducted by Popat [43] including 442 cases confirmed that POI patients have a lower BMD compared to regularly menstruating women. According to a study by Nelson et al. [44] $67 \%$ of patients with POI have osteopenia. Leite-Silva et al. [45] studied 50 women with POI and found a decrease in the lumbar spine and femoral BMD. The lumbar part of the spine was the most affected by the BMD decrease. They reported that age generally, age of POI and reproductive age were factors associated with the BMD of the lumbar spine.

Total body BMD clearly corresponds to the duration of ovarian function in POI patients [46].

There are limited data regarding fracture risk in POI patients. Clinical studies that compared women experiencing menopause at normal age to women who had premature menopause reported relative risks for fracture of approximately 1.5 in women with premature menopause [47].

\section{Autoimmune and thyroid disease risk}

It is estimated that $4-30 \%$ of POI cases are due to autoimmune aetiology. Therefore, the autoimmune diseases risk is increased in this group [48]. According to available data, the incidence of autoimmune hypothyroidism, adrenal insufficiency, diabetes type 1, hypoparathyroidism and pernicious anaemia is increased [49].

Autoimmune thyroiditis seems to be the most common autoimmune disease in the POI population. Up to $24 \%$ of POI women were reported positive for thyroid peroxidase autoantibodies [50]. The overt hypothyroidism is present in about $8-20 \%$ of POI individuals [51, 52]. 21-hydroxylase adrenal autoantibodies have been reported in $3.2 \%$ of POI women. Similarly, approximately $2-3 \%$ of women will have asymptomatic autoimmune adrenal insufficiency [52, 53]. According to another report, women with adrenal autoimmunity detected by the presence of autoantibodies have a $50 \%$ risk of developing adrenal insufficiency [53].

The incidence of type 1 diabetes in POI, according to available reports, is $2.5 \%$ [54]. The reports about the association of POI with other autoimmune diseases are anecdotal; therefore, these disorders are relatively uncommon.

It has been also shown that non-autoimmune POI can be either linked to increased risk of developing autoimmune disease. In Nurses Health Study, early menopause (including surgical) early menopause (at age $<45$ years) has been related to augmented risk of systemic lupus erythematosus and rheumatoid arthritis [54]. The pathophysiological mechanisms related to these phenomena seem to be complex. Probably the immunomodulatory effects of estradiol (especially on $\mathrm{T}$ helper cells) are of importance, but this issue requires further evaluation [55].

\section{Cognitive dysfunction}

Work by several groups has provided evidence that early menopause can be associated with neurological dysfunction and an increased risk of dementia. The data suggest an increased risk of neurological disorder where POI is due to premature menopause or induced from surgery. This increased risk appears to be most apparent in the domains of global cognitive and verbal memory tests. Where POI is caused by genetic disorder, observed cognitive deficiencies may be more likely to have a genetic basis rather than being due to the effects of sex steroids on the brain. Findings related to the loss of cognitive function after chemotherapy or GnRH analogues treatments are mixed. 
Nappi et al. [56] investigated attentive and verbal memory performances in physiological and surgical menopause, drawing attention to the impact of age at menopause. Surgical menopause affects short-term verbal memory more than physiological menopause.

A recent publication of Bove et al. [57] determined the association between age at surgical menopause and both cognitive decline and Alzheimer's disease in two longitudinal cohorts. The researchers found that early age at surgical menopause was associated with cognitive decline and the incidence of Alzheimer's disease.

In a longitudinal study, Farrag et al. [58] investigated the effect of oestrogen deficiency on cognitive function in surgically menopausal women $(n=35)$. Rapid decline in the oestrogen level following surgical menopause was associated with a deleterious effect on cognitive function.

Another study from the Mayo Clinic Cohort Study of Oophorectomy and Ageing, investigated women who had both ovaries removed before reaching natural menopause. The authors found that examined women experienced a long-term increased risk of Parkinsonism, cognitive impairment or dementia, depressive and anxiety symptoms [59].

Phung et al. [60] revealed that premenopausal bilateral oophorectomy is associated with a higher risk of dementia, suggesting a dose effect of premature oestrogen deficiency on dementia. The age-dependent effect suggests that the younger brain is more vulnerable to oestrogen deficiency.

\section{Mood and sexual disorders}

The psychosocial aspects of POI are most often ignored in the context of the diagnosis. The decrease in the mood can be caused not only by concerns about their own health, but also reproductive problems that occur in younger women who want to have children. Being diagnosed with POI can be an unexpected and upsetting diagnosis. Women with POI experience significant psychologic disturbances, such as high levels of depression and low levels of self-esteem, with negative effects on sexuality [61]. The diagnosis of POI can be an extremely devastating life experience and patients often express anger, depression, anxiety, loss and sadness. Women after being informed of POI diagnosis can be shocked and confused. These words are describing their emotional trauma. Some of POI patients report a range of emotions and providers should offer support regarding the patient's altered self-image, sexual dysfunction and neurocognitive decline.

Schmitdt et al. [62] evaluated depression in women with spontaneous 46, XX primary ovarian insufficiency. The researchers compared prevalence of depressive symptoms observed in women with primary ovarian insufficiency (POI) with women in whom the menopause is normally timed. They studied 174 women with spontaneous $46, \mathrm{XX}$ POI and 100 women with Turner syndrome. POI proved to be associated with an increased lifetime risk for major depression. The scientists stressed that more attention should be paid to the presence of depression in POI. Moreover, the onset of depression frequently occured after signs of altered ovarian function but before the diagnosis of POI.

Fang et al. [63] prospectively compared quality of life (QOL) in 75 women at increased risk of ovarian cancer who are undergoing risk-reducing salpingo-oophorectomy. Women who underwent surgery revealed poorer physical functioning, greater pain, less vitality, decreased social functioning, greater discomfort and less satisfaction with sexual activities at 1 month compared to controls. However, most QOL deficits observed in the surgical group were no longer apparent by the 6-month assessment.

A cross-sectional study comparing sexual function of 58 POI patients with eumenorrheic women revealed that POI patients experienced worse sexual function in relation to satisfaction, lubrication, orgasm, pain and arousal. However, there were no differences between the two groups with respect to desire [64]. Similar results showed another study including 81 POI patients complaining of less sexual arousal, reduced lubrication and increased genital pain, without a disturbed feeling of desire comparing to healthy controls [65]. The POI patients in this study had decreased serum androgen serum levels in comparison to the control group. Detailed analysis of the hormonal profiles of the study group revealed the weak influence of androgen on sexual functioning. Higher total testosterone levels were associated with an increased frequency of desire, and higher androstenedione levels were associated with an elevated frequency of sexual contact [67].

Dennerstein et al. [66] determined the prevalence of hypoactive sexual desire disorder (HSDD) among women who have undergone surgical menopause with that of premenopausal or naturally menopausal women. The surgical menopausal women are at an increased risk for HSDD. HSDD is associated with diminished sexual and partner relationship satisfaction and negative emotional states.

\section{Mortality}

Overall mortality is increased in women with POI. According to data from a cohort of 19,731 women from Norway found that the menopause before 40 years old was linked to an increased mortality rate of 1.06 (CI 0.99-1.14) in comparison to women who had menopause at the age 50-52 [67]. Also early menopause has been shown to reduce the life expectancy. Data from a prospective cohort study of 68,154 US adult women showed that all-cause mortality rates were higher among women who reported that their 
menopause occurred at age 40-44 years compared with women who reported that their menopause occurred at age $50-54$ years [rate ratio $(\mathrm{RR})=1.04,95 \%$ confidence interval (CI) $1.00,1.08]$ [68].

The increased risk of all-cause mortality is mainly dependent on higher mortality rates from coronary heart disease $(\mathrm{RR}=1.09,95 \%$ CI $1.00,1.18)$, respiratory disease $(\mathrm{RR}=1.19,95 \% \mathrm{CI} 1.02,1.39)$, genitourinary disease $(\mathrm{RR}=1.39,95 \% \mathrm{CI} 1.07,1.82)$ and external causes $(\mathrm{RR}=1.56,95 \% \mathrm{CI} 1.21,2.02)[69]$.

\section{Summary: clinical implications}

POI patients have an increased risk of certain morbidity as well as mortality, as shown above. Recently, the European Society of Human Reproduction published management guidelines for POI [69]. According to expert opinion women with POI should be advised on how to reduce cardiovascular risk factors by not smoking, by taking regular exercise and maintaining a healthy weight to reduce the risk of premature death. The cardiovascular evaluation should consist of monitoring annually blood pressure, weight and smoking status. As we have stated above, the chance for spontaneous pregnancy is very low; therefore, oocyte donation is the best option for fertility. The risk of BMD decrease and osteoporosis requires proper management, i.e. lifestyle interventions, sufficient intake of calcium and vitamin D and hormonal replacement therapy. All the patients diagnosed with POI should be evaluated for BMD (densitometry). Due to the risk of psychological problems, patients should be allowed to obtain psychological help. Sexual dysfunction should be managed by proper counselling, oestrogen replacement and androgen supplementation in chosen cases. Vaginal atrophy may by improved by the use of topical oestrogens and lubricants. According to expert opinion, the neurological well-being should be supported by healthy lifestyle interventions and oestrogen replacement.

\section{Compliance with ethical standards}

Conflict of interest The authors do not declare any conflict of interest.

\section{Ethical approval Not applicable.}

Informed consent Not applicable.

Funding This research did not receive any specific Grant from any funding agency in the public, commercial, or not-for-profit sector.

Open Access This article is distributed under the terms of the Creative Commons Attribution 4.0 International License (http://creativecommons.org/licenses/by/4.0/), which permits unrestricted use, distribution, and reproduction in any medium, provided you give appropriate credit to the original author(s) and the source, provide a link to the Creative Commons license, and indicate if changes were made.

\section{References}

1. Luisi S, Orlandini C, Regini C, Pizzo A, Vellucci F, Petraglia F (2015) Premature ovarian insufficiency: from pathogenesis to clinical management. J Endocrinol Invest 38(6):597-603

2. Vujovic S, Brincat M, Erel T, Gambacciani M, Lambrinoudaki I, Moen MH, Schenck-Gustafsson K, Tremollieres F, Rozenberg S, Rees M (2010) EMAS position statement: managing women with premature ovarian failure. European Menopause and Andropause Society. Maturitas 67(1):91-93

3. Coulam CB, Adamson SC, Annegers JF (1986) Incidence of premature ovarian failure. Obstet Gynecol 67(4):604-606

4. Shuster LT, Rhodes DJ, Gostout BS, Grossardt BR, Rocca WA (2010) Premature menopause or early menopause: long-term health consequences. Maturitas 65(2):161-166

5. Albright F, Smith PH, Fraser R (1942) A syndrome characterized by primary ovarian insufficiency and decreased stature: report of 11 cases with a digression on hormonal control of axillary and pubic hair. Am J Med Sci 204:625-648

6. Nelson LM (2009) Clinical practice. Primary ovarian insufficiency. N Engl J Med 360:606-614

7. Nuovo S, Passeri M, Di Benedetto E, Calanchini M, Meldolesi I, Di Giacomo MC, Petruzzi D, Piemontese M, Zelante L, Sangiuolo F, Novelli G, Fabbri A, Brancati F (2016) Characterization of endocrine features and genotype-phenotypes correlations in blepharophimosis-ptosis-epicanthus inversus syndrome type 1 . J Endocrinol Invest 39(2):227-233

8. Chang SH, Kim CS, Lee KS, Kim H, Yim SV, Lim YJ, Park SK (2007) Premenopausal factors influencing premature ovarian failure and early menopause. Maturitas 58(1):19-30

9. Wang H, Chen H, Qin Y, Shi Z, Zhao X, Xu J, Ma B, Chen ZJ (2015) Risks associated with premature ovarian failure in Han Chinese women. Reprod Biomed Online 30(4):401-407

10. Lokkegaard E, Jovanovic Z, Heitmann BL, Keiding N, Ottesen B, Pedersen AT (2006) The association between early menopause and risk of ischaemic heart disease: influence of hormone therapy. Maturitas 53:226-233

11. Bidet M, Bachelot A, Bissauge EE, Golmard JL, Gricout S, Dulon J, Coussieu C, Badachi Y, Touraine P (2011) Resumption of ovarian function and pregnancies in 358 patients with premature ovarian failure. J Clin Endocrinl Metab 96:3864-3872

12. Sassarini J, Lumdsen MA, Critchley HOD (2015) Sex hormone replacement in ovarian failure-new treatment concepts. Best Pract Res Clin Endocrinol Metabol 29:105-114

13. Robles A, Checa MA, Prat M, Carreras R (2013) Medical alternatives to oocyte donation in women with premature ovarian failure: a systematic review. Gyn Endocrinol 29:632-637

14. Naredi N, Sandeep K, Jamwal VDS (2013) Can hormone replacement therapy prior to oocyte donation cycle in women with premature ovarian failure improve pregnancy rate? Med J Armed Forces India 69:357-360

15. Ameratunga D, Weston G, Osiankis T, Catt J, Vollenhoven B (2009) In vitro fertilization (IVF) with donor eggs in post-menopausal women: are there differences in pregnancy outcomes in women with premature ovarian failure (POF) compared with women with physiological age-related menopause? J Assist Reprod Genet 26:511-514

16. Del Mastro L, Ceppi M, Poggio F, Bighin C, Peccatori F, Demeestere I, Levaggi A, Giraudi S, Lambertini M, D’Alonzo A, 
Canavese G, Pronzato P, Bruzzi P (2014) Gonadotropin-releasing hormone analogues for the prevention of chemotherapyinduced premature ovarian failure in cancer women: systematic review and meta-analysis of randomized trials. Cancer Treat Rev 40:675-683

17. Sun X, Dongol S, Jiang J, Kong B (2014) Protection of ovarian function by GnRH agonists during chemotherapy: a meta-analysis. Int J Oncol 44:1335-1340

18. Rodriguez-Wallber KA, Otkay K (2012) Options on fertility preservation in female cancer patients. Cancer Treat Rev 38:354-361

19. Kasum M, Beketic-Oreskovic L, Peddi PF, Oreskovic S, Johnson RH (2014) Fertility after breast cancer treatment. Eur J Obstet Gynecol Reprod Biol 173:13-18

20. Portman DJ, Ml Gass (2014) Genitourinary syndrome of menopause: new terminology for vulvovaginal atrophy form the International Society for the Study of Women's Sexual Health and the North American Menopause Society. J Sex Med 11:2865-2872

21. Gibson-Helm M, Teede H, Vincent A (2014) Symptoms, health behavior and understanding of menopause therapy in women with premature menopause. Climacteric 17:666-673

22. Benetti-Pinto CL, Giraldo PC, Pacello PC, Soares PM, Yela DA (2015) Vaginal epithelium and microflora characteristics in women with premature ovarian failure under hormone therapy compared to healthy women. Arch Gynecol Obstet 92:159-164

23. Pacello PC, Yela DA, Rabelo S, Giraldo PC, Benetti-Pinto CL (2014) Dyspareunia and lubrication in premature ovarian failure using hormonal therapy and vaginal health. Climacteric 17:342-347

24. Karram MM, Yeko TR, Sauer MV, Bhatia NN (1989) Urodynamic changes following hormonal replacement therapy in women with premature ovarian failure. Obstet Gynecol 74:208-211

25. Ewertz M, Mellemkjaer I, Poulsen AH (2005) Hormone use for menopausal symptoms and risk of breast cancer. A Danish cohort study. Br J Cancer 92:1293-1297

26. Mendoza N, Julia D, Galliano D, Coronado P, Diaz B, Fontez J, Gallo JL, Garcia A, Guinot M, Munnamy M, Roca B, Sosa M, Tomas J, Llaneza P, Sanchez-Borrego R (2015) Spanish consensus on premature menopause. Maturitas 80:220-225

27. Hershlag A, Raush ME, Cohen M (2011) Ovarian failure in adolescent cancer survivors should be treated. J Pediatr Adolesc Gynecol 24:101-103

28. Fish JD (2011) Hormone replacement for survivors of childhood cancer with ovarian failure-when is it worth the risk? J Pediatr Adolesc Gynecol 24:98-101

29. Wu X, Cai H, Kallianpur A, Li H, Yang G, Gao J, Xiang Y-B, Ji B-T, Zheng W, Shu X-O (2014) Impact of premature ovarian failure on mortality and morbidity among Chinese women. PLoS One 9:e89597. doi:10.1371/journal.pone.0089597

30. Yorgun H, Tokgözoğlu L, Canpolat U, Gürses KM, Bozdağ G, Yapıcı Z, Sahiner L, Kaya EB, Kabakçı G, Oto A, Tuncer M, Aytemir K (2013) The cardiovascular effects of premature ovarian failure. Int J Cardiol 168(1):506-510

31. Kalantaridou SN, Naka KK, Papanikolaou E, Kazakos N, Kravariti M, Calis KA, Paraskevaidis EA, Sideris DA, Tsatsoulis A, Chrousos GP, Michalis LK (2004) Impaired endothelial function in young women with premature ovarian failure: normalization with hormone therapy. J Clin Endocrinol Metab 89(8):3907-3913

32. Goldmeier S, De Angelis K, Rabello Casali K, Vilodre C, Consolim-Colombo F, Belló Klein A, Plentz R, Spritzer P, Irigoyen MC (2013) Cardiovascular autonomic dysfunction in primary ovarian insufficiency: clinical and experimental evidence. Am J Transl Res 6(1):91-101

33. Knauff EA, Westerveld HE, Goverde AJ, Eijkemans MJ, Valkenburg O, van Santbrink EJ, Fauser BC, van der Schouw YT (2008)
Lipid profile of women with premature ovarian failure. Menopause 15(5):919-923

34. Gulhan I, Bozkaya G, Uyar I, Oztekin D, Pamuk BO, Dogan E (2012) Serum lipid levels in women with premature ovarian failure. Menopause 19(11):1231-1234

35. Ates S, Yesil G, Sevket O, Molla T, Yildiz S (2014) Comparison of metabolic profile and abdominal fat distribution between karyotypically normal women with premature ovarian insufficiency and age matched controls. Maturitas 79(3):306-310

36. Kulaksizoglu M, Ipekci SH, Kebapcilar L, Kebapcilar AG, Korkmaz H, Akyurek F, Baldane S, Gonen MS (2013) Risk factors for diabetes mellitus in women with primary ovarian insufficiency. Biol Trace Elem Res 154(3):313-320

37. Jacobsen BK, Knutsen SF, Fraser GE (1999) Age at natural menopause and total mortality and mortality from ischemic heart disease: the Adventist Health Study. J Clin Epidemiol 52:303-307

38. Schnatz PF (2011) The 2010 North American Menopause Society position statement: updates on screening, prevention and management of postmenopausal osteoporosis. Conn Med 75(8):485-487

39. Albright F, Smith P, Richardson AM (1941) Post menopausal osteoporosis: its clinical features. JAMA 116:2465-2474

40. Meczekalski B, Podfigurna-Stopa A, Genazzani AR (2010) Hypoestrogenism in young women and its influence on bone mass density. Gynecol Endocrinol 26(9):652-657

41. Lana MB, Straminsky V, Onetto C, Amuchastegui JM, Blanco G, Galluzzo L, Provenzano S, Nolting M (2010) What is really responsible for bone loss in spontaneous premature ovarian failure? A new enigma. Gynecol Endocrinol 26(10):755-759

42. Uygur D, Sengul O, Bayar D, Erdinc S, Batioglu S, Mollamahmutoglu L (2005) Bone loss in young women with premature ovarian failure. Arch Gynecol Obstet 273:17-19

43. Popat VB, Calis KA, Vanderhoof VH, Cizza G, Reynolds JC, Sebring N, Troendle JF, Nelson LM (2009) Bone mineral density in estrogen-deficient young women. J Clin Endocrinol Metab 94(7):2277-2283

44. Nelson LM, Convington SN, Rebar RW (2005) An update: spontaneous premature ovarian failure is not a menopause. Fertil Steril 83:1327-1332

45. Leite-Silva P, Bedone A, Pinto-Neto AM, Costa JV, Costa-Paiva L (2009) Factors associated with bone density in young women with karyotypically normal spontaneous premature ovarian failure. Arch Gynecol Obstet 280(2):177-181

46. Bakhsh H, Dei M, Bucciantini S, Balzi D, Bruni V (2015) Premature ovarian insufficiency in young girls: repercussions on uterine volume and bone mineral density. Gynecol Endocrinol 31(1):65-69

47. van Der Voort DJ, van Der Weijer PH, Barentsen R (2003) Early menopause: increased fracture risk at older age. Osteoporos Int 14(6):525-530

48. Bakalov VK, Anasti JN, Calis KA, Vanderhoof VH, Premkumar A, Chen S, Furmaniak J, Smith BR, Merino MJ, Nelson LM (2005) Autoimmune oophoritis as a mechanism of follicular dysfunction in women with 46, XX spontaneous premature ovarian failure. Fertil Steril 84(4):958-965

49. Yan G, Schoenfeld D, Penney C, Hurxthal K, Taylor AE, Faustman D (2000) Identification of premature ovarian failure patients with underlying autoimmunity. J Womens Health Gend Based Med 9(3):275-287

50. Goswami R, Marwaha RK, Goswami D, Gupta N, Ray D, Tomar N, Singh S (2006) Prevalence of thyroid autoimmunity in sporadic idiopathic hypoparathyroidism in comparison to type 1 diabetes and premature ovarian failure. J Clin Endocrinol Metab 91(11):4256-4259

51. Kim TJ, Anasti JN, Flack MR, Kimzey LM, Defensor RA, Nelson LM (1997) Routine endocrine screening for patients with 
karyotypically normal spontaneous premature ovarian failure. Obstet Gynecol 89(5 Pt 1):777-779

52. Bakalov VK, Vanderhoof VH, Bondy CA, Nelson LM (2002) Adrenal antibodies detect asymptomatic auto-immune adrenal insufficiency in young women with spontaneous premature ovarian failure. Hum Reprod 17(8):2096-2100

53. Betterle C, Volpato M, Rees Smith B, Furmaniak J, Chen S, Greggio NA, Sanzari M, Tedesco F, Pedini B, Boscaro M, Presotto F (1997) Adrenal cortex and steroid 21-hydroxylase autoantibodies in adult patients with organ-specific autoimmune diseases: markers of low progression to clinical Addison's disease. J Clin Endocrinol Metab 82(3):932-938

54. Costenbader KH, Feskanich D, Stampfer MJ, Karlson EW (2007) Reproductive and menopausal factors and risk of systemic lupus erythematosus in women. Arthritis Rheum 56:1251-1262

55. Bove R (2013) Autoimmune diseases and reproductive aging. Clin Immunol 149(2):251-264

56. Nappi RE, Sinforiani E, Mauri M, Bono G, Polatti F, Nappi G (1999) Memory functioning at menopause: impact of age in ovariectomized women. Gynecol Obstet Invest 47:29-36

57. Bove R, Secor E, Chibnik LB, Barnes LL, Schneider JA, Bennett DA, De Jager PL (2014) Age at surgical menopause influences cognitive decline and Alzheimer pathology in older women. Neurology 82(3):222-229

58. Farrag AK, Khedr EM, Abdel-Aleem H, Rageh TA (2002) Effect of surgical menopause on cognitive functions. Dement Geriatr Cogn Disord 13:193-198

59. Rocca WA, Shuster LT, Grossardt BR, Maraganore DM, Gostout BS, Geda YE, Melton LJ 3rd (2009) Long-term effects of bilateral oophorectomy on brain aging: unanswered questions from the Mayo Clinic Cohort Study of Oophorectomy and Aging. Womens Health (Lond Engl) 5(1):39-48

60. Phung TK, Waltoft BL, Laursen TM, Settnes A, Kessing LV, Mortensen PB, Waldemar G (2010) Hysterectomy, oophorectomy and risk of dementia: a nationwide historical cohort study. Dement Geriatr Cogn Disord 30(1):43-50
61. Schmidt PJ, Cardoso GM, Ross JL, Haq N, Rubinow DR, Bondy CA (2006) Shyness, social anxiety, and impaired self-esteem in Turner syndrome and premature ovarian failure. JAMA 295:1374-1376

62. Schmidt PJ, Luff JA, Haq NA, Vanderhoof VH, Koziol DE, Calis KA, Rubinow DR, Nelson LM (2011) 0 Depression in women with spontaneous 46 , XX primary ovarian insufficiency. J Clin Endocrinol Metab 96(2):E278-E287

63. Fang CY, Cherry C, Devarajan K, Li T, Malick J, Daly MB (2009) A prospective study of quality of life among women undergoing risk-reducing salpingo-oophorectomy versus gynecologic screening for ovarian cancer. Gynecol Oncol 112(3):594-600

64. de Almeida DM, Benetti-Pinto CL, Makuch MY (2011) Sexual function of women with premature ovarian failure. Menopause $18: 262-266$

65. van der Stege JG, Groen H, van Zadelhoff SJ, Lambalk CB, Braat DD, van Kastern YM, van Santbrink EJ, Apperloo MJ, Weijmar Schultz WC, Hoek A (2008) Decreased androgen concentrations and diminished general and sexual well-being in women with premature ovarian failure. Menopause 15:23-31

66. Dennerstein L, Koochaki P, Barton I, Graziottin A (2006) Hypoactive sexual desire disorder in menopausal women: a survey of Western European women. J Sex Med 3(2):212-222

67. Jacobsen BK, Heuch I, Kvåle G (2003) Age at natural menopause and all-cause mortality: a 37-year follow-up of 19,731 Norwegian women. Am J Epidemiol 157(10):923-929

68. Mondul AM, Rodriguez C, Jacobs EJ, Calle EE (2005) Age at natural menopause and cause-specific mortality. Am J Epidemiol 162(11):1089-1097

69. POI Guideline Development Group (2015) Management of women with premature ovarian insufficiency. Guideline of the European Society of Human Reproduction and Embryology. European Society of Human Reproduction and Embryology, Belgium 